\title{
Transvaginal ultrasound guided trigone and bladder injection: A cadaveric feasibility study for a novel route of intradetrusor chemodenervation
}

\author{
Raveen Syan ${ }^{1}$, Mason A. Briggs ${ }^{1}$, John C. Olivas ${ }^{2}$, Sakti Srivastava ${ }^{2}$, Craig V. Comiter ${ }^{1}$, Amy D. Dobberfuhl ${ }^{1}$ \\ Departments of'Urology, ${ }^{2}$ Surgery, Stanford University School of Medicine, Stanford, CA, USA
}

Purpose: OnabotulinumtoxinA (BTX) detrusor chemodenervation is an efficacious third-line treatment for overactive bladder. Despite high clinical efficacy rates for BTX injection, many patients refuse initial or repeat treatment due to the invasiveness of the cystoscopic route of delivery. We assess the feasibility of injecting the trigone and posterior bladder wall via a transvaginal route under ultrasound guidance using a human cadaveric model.

Materials and Methods: Eight de-identified anonymous fresh female deceased donor cadaver pelvises were placed in supine split leg position. A transvaginal ultrasound probe guided injections of India ink into the trigone in 3 sites and the posterior wall in 2 sites. Full thickness bladder biopsies were then obtained and histologic analysis was performed to confirm presence of India ink in the detrusor layer.

Results: The mean time from day of death was 11.0 days (range, 4.0-23.0 days). Three to five bladder biopsies were obtained per cadaver, for a total of 34 specimens (20 trigone, 14 posterior wall). Histologic analysis revealed presence of India ink within the detrusor layer in 8/8 (100.0\%) of cadavers. The surgeon's perception of appropriate targeting under ultrasound guidance was confirmed in $8 / 8$ cadavers (100.0\%) involving the bladder trigone, and $7 / 8$ (87.5\%) involving the posterior wall. Of injections that were believed to have appropriately targeted the detrusor layer, 22/34 specimens (64.7\%) demonstrated the presence of India ink under histologic analysis.

Conclusions: Intradetrusor injection of the bladder trigone and posterior wall under transvaginal ultrasound guidance is feasible and has acceptable accuracy.

Keywords: Botulinum A toxin; Cadaver; Intravesical injection; Ultrasound imaging; Urinary bladder, overactive

This is an Open Access article distributed under the terms of the Creative Commons Attribution Non-Commercial License (http://creativecommons.org/licenses/by-nc/4.0) which permits unrestricted non-commercial use, distribution, and reproduction in any medium, provided the original work is properly cited.

\section{INTRODUCTION}

Up to 1 in 5 adult men and women will be affected by overactive bladder $(\mathrm{OAB})$ at some point in their lifetime. Given the high disease burden and reported prevalence of $12 \%$ to $19 \%$, this condition is frequently encountered in clinical practice and results in a marked reduction in quality of life when inadequately treated [1]. The American Urological Association and Society of Urodynamics, Female Pelvic Medicine and Urogenital Reconstruction Guidelines

Received: 21 September, 2018 - Accepted: 7 December, 2018

Corresponding Author: Raveen Syan

Department of Urology, Stanford University School of Medicine, 300 Pasteur Drive, Grant S-287, Stanford, CA 94305, USA

TEL: +1-650-725-5746, FAX: +1-650-498-5346, E-mail: rsyan@stanford.edu

ORCID: https://orcid.org/0000-0002-4020-8004 
for the management of $\mathrm{OAB}$ recommend onabotulinumtoxinA (BTX) intradetrusor chemodenervation and neuromodulation as third-line treatment for those who fail behavioral and pharmacological therapy [2] The safety and efficacy of chemodenervation has been demonstrated by several randomized controlled clinical trials, with $65 \%$ of patients reporting significant improvement in symptoms [3].

Interestingly, a number of studies have shown that inclusion of BTX injection into the trigone of the bladder, either alone, or in conjunction with the standard injection template with emphasis on the posterior-lateral walls of the bladder, may provide adequate improvement in lower urinary tract symptoms for many patients [4-9]. Dobberfuhl et al. [8] demonstrated in a rat model that there were no adverse changes in ambulatory voiding function after trigone BTX detrusor injection. Similarly in the human, Pinto et al. [10] demonstrated that isolated cystometric injection of the bladder trigone for patients with interstitial cystitis/bladder pain syndrome resulted in improvements in OAB-related urinary frequency at 1, 2, and 3 months when compared to baseline.

One barrier to intradetrusor BTX injection is the requirement for cystoscopy in order to inject the walls of the bladder. This procedure is invasive, and requires equipment turnover, nursing assistance, and sterilization of the cystoscope prior to reuse. This process carries with its significant equipment costs to deliver BTX to the bladder detrusor muscle. In addition, the passage of the cystoscope through the urethra can be quite uncomfortable, with $15 \%$ of patients developing urethrorrhagia and dysuria following cystoscopy [11], and a $22 \%$ rate of urinary tract infection after BTX injection [1]. Despite obvious clinical efficacy of BTX chemodenervation, it is not uncommon for patients to cease therapy beyond their first injection simply to avoid repeat cystoscopy. Long term compliance with BTX therapy has been shown to be as low as $25 \%$, where $77 \%$ of patients who discontinued therapy reported problems related to tolerability of the intervention [12]. The aim of our study is to develop a novel and more facile technique to deliver BTX therapy to the bladder trigone and posterior wall using a transvaginal ultrasound-guided approach. We use a cadaveric model to demonstrate this novel delivery technique by injecting fresh cadavers transvaginally under ultrasound guidance in this pre-clinical feasibility trial.

\section{MATERIALS AND METHODS}

We performed a cadaveric proof of concept study using eight de-identified anonymous fresh female deceased donor cadaver pelvises. Deceased de-identified anonymous cadaver tissue research is exempt from Institutional Review Board living human subject research at Stanford University. Our cadaver procedural protocol was approved by the division of clinical anatomy. Fresh cadavers met our inclusion criteria of being female gender, having a patent vagina, and no prior pelvic surgery. The cadaver was placed in a supine split leg position. A 16 French Foley catheter was placed in the bladder; the bladder was irrigated clear and then filled with $300 \mathrm{~mL}$ of water. With the bladder distended and palpable, we placed a suprapubic laparoscopic trochar into the bladder dome. A zero degree lens was used to visualize the bladder trigone and ureteral orifices through the laparoscopic trochar. Next, a transvaginal ultrasound probe, approved for use in cadavers, was placed in the vagina. Anatomic landmarks were measured using ultrasound, including bladder neck thickness, posterior wall thickness, and interureteric distance. Using the Foley balloon to localize the bladder neck and trigone under ultrasound guidance, an 18 gauge, $25 \mathrm{~cm}$ needle was directed into the bladder trigone. The laparoscope confirmed appropriate targeting of the trigone (Fig. 1). The combination of the laparoscopic view and ultrasound guidance confirmed that the position of the ureteral orifices were identifiable via transvaginal ultrasound. We injected a total of $3 \mathrm{~mL}$ of dilute blue India ink (3 drops ink per $10 \mathrm{~mL}$ saline) under ultrasound guidance divided into three locations along the bladder trigone (1 $\mathrm{mL}$ per injection). Ultrasound images pre and post-injection were saved (Fig. 1). We then injected $3 \mathrm{~mL}$ of dilute green India ink (3 drops ink per $10 \mathrm{~mL}$ saline), divided into two locations along the posterior bladder wall under ultrasound guidance ( $1.5 \mathrm{~mL}$ per injection). There was no dye extravasation into the lumen of the bladder on cystoscopy.

Once injection was completed, a suprapubic cystotomy was performed and gross visualization of the location of the two dye colors was used to confirm if ultrasound guided targeting of the trigone and posterior bladder wall was accurate (Fig. 1). Dissection confirmed there was no interposing bowel along the most apical extent of our injection in all cadavers. Full thickness bladder and vaginal wall tissue was obtained for histology using an $8 \mathrm{~mm}$ punch biopsy from the midline trigone, ureteral orifice, posterior wall and lateral wall in order to co-localize depth and diffusion of injection with muscle and nerve fibers. Tissue was fixed in formalin, paraffin embedded, mounted onto slides, and stained with Hematoxylin and Eosin. Slides were then semi-qualitatively assessed for the presence and location of India ink, while blinded to intra-procedure findings. 

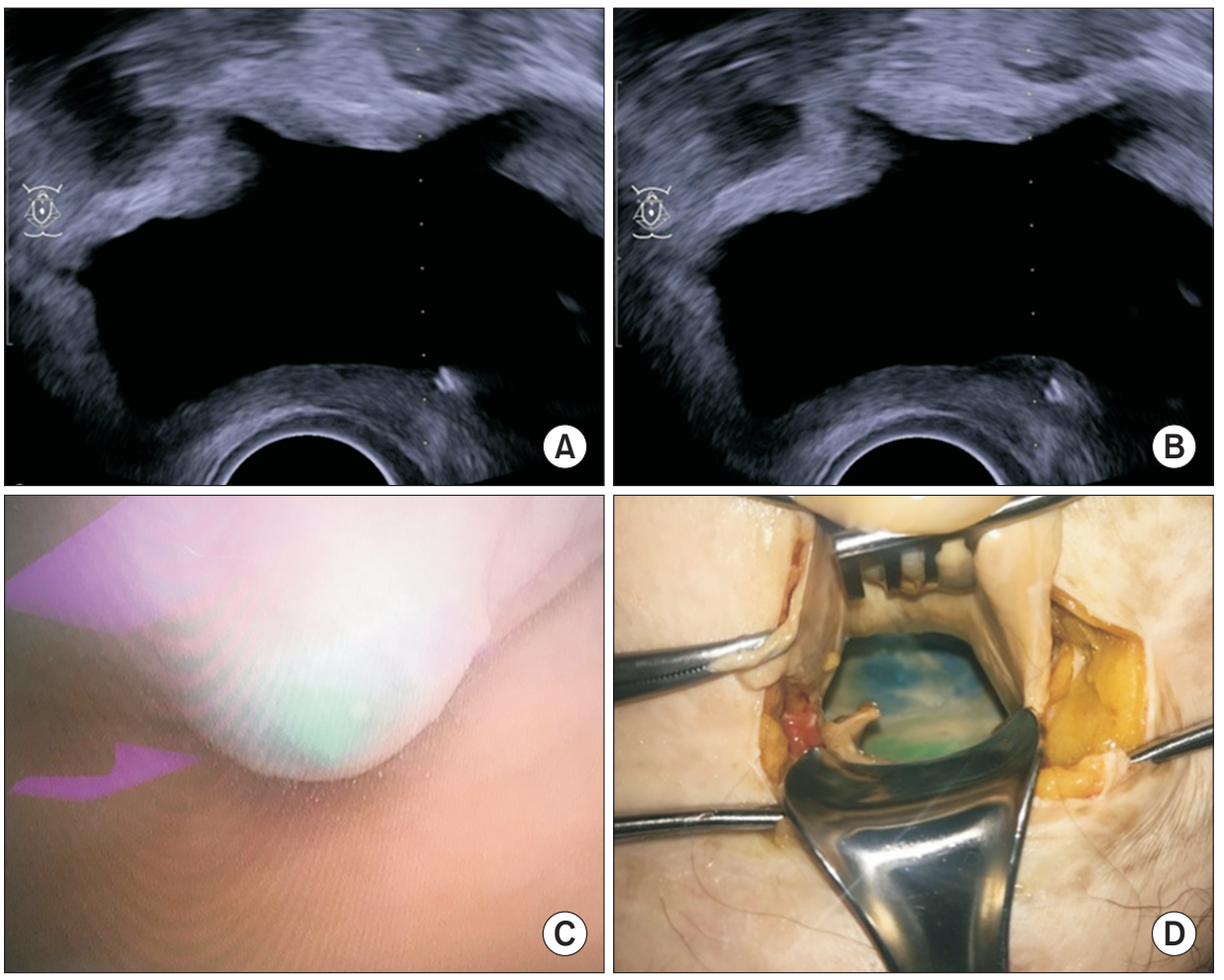

Fig. 1. Ultrasound, cystoscopic and gross visualization of the bladder trigone. (A) Ultrasound pre-injection. (B) Ultrasound post-injection. (C) Cystoscopy pre-injection. (D) Cystotomy post-injection.

Table 1. Cadaver characteristics and anatomic variability

\begin{tabular}{lcc}
\hline \multicolumn{1}{c}{ Cadaveric characteristic } & Mean & Range \\
\hline Interval between date of death and date of procedure $(\mathrm{d})$ & 11.0 & $4.0-23.0$ \\
Interureteric distance $(\mathrm{mm})$ & 24.4 & $20.2-32.3$ \\
Bladder neck thickness $(\mathrm{mm})$ & 8.2 & $5.4-11.2$ \\
Posterior wall thickness $(\mathrm{mm})$ & 4.0 & $3.1-4.9$ \\
\hline
\end{tabular}

\section{RESULTS}

Eight fresh cadavers were utilized in the study period that met our criteria. The mean time from day of death was 11.0 days (range, 4.0-23.0 days). Interureteric distance measured by ultrasound and was similar amongst cadavers with a mean distance of $24.4 \mathrm{~mm}$ (range, $20.2-32.3 \mathrm{~mm}$ ), as was posterior wall thickness (mean, $4.0 \mathrm{~mm}$; range, 3.1-4.9 $\mathrm{mm}$ ), while bladder neck thickness (mean, $8.2 \mathrm{~mm}$; range, 5.4-11.2 $\mathrm{mm}$ ) demonstrated variability between cadavers (Table 1). Three to five bladder biopsies were obtained per cadaver, for a total of 34 specimens (20 trigone, 14 posterior wall). Histologic analysis revealed the presence of India ink within the detrusor and/or suburothelial layer in $8 / 8$ $(100.0 \%)$ of cadavers. Presence of co-localized green and blue India ink was evident in the trigone and/or posterior walls in $4 / 8$ (50.0\%) of cadavers, indicating diffusion of ink following injection (Fig. 2). The surgeon's perception of appropriate targeting under ultrasound guidance was $8 / 8$ cadavers (100.0\%) involving the bladder trigone, and $7 / 8$
(87.5\%) involving the posterior wall (one cadaver had severe intrinsic sphincter deficiency, limiting bladder distension and thereby posterior wall localization). By specimen, of injections that were believed to have appropriately targeted the detrusor layer, 22/34 specimens (64.7\%) demonstrated the presence of India ink under histologic analysis (Fig. 3).

\section{DISCUSSION}

Our study confirms that transvaginal injection under ultrasound guidance is feasible in the female cadaver with acceptable accuracy. By avoiding entry into the bladder lumen, we hypothesize that we will see a reduction in number of urinary tract infections and discomfort related to the cystoscopic route when performing transvaginal ultrasound guided intradetrusor injection in live humans $[1,11]$. The tolerability of transvaginal trigger point injection for management of pelvic floor disorders is well-established [13]. The bladder trigone contains the highest density of autonomic nerves in women [14], therefore by specifically 
Intradetrusor and suburothelial India ink present

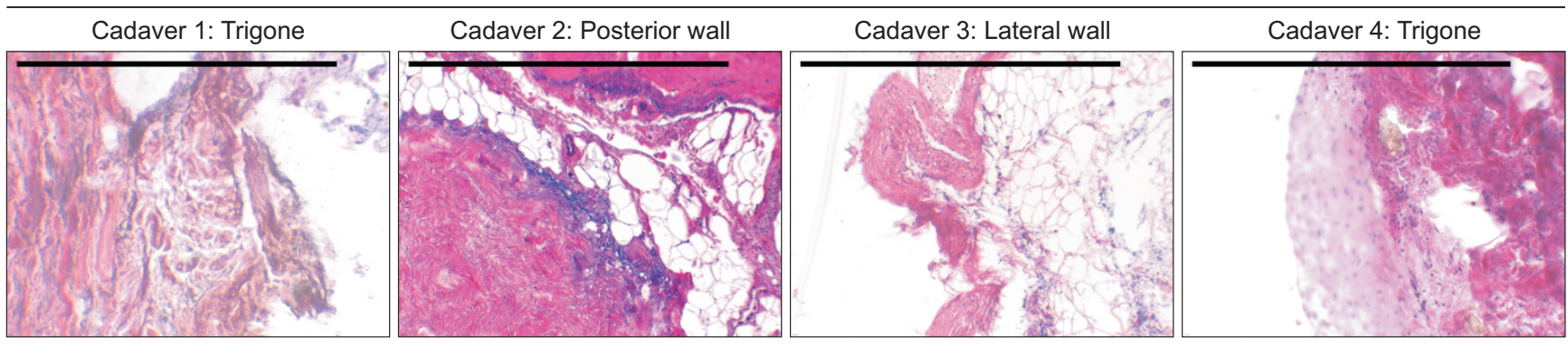

Colocalization of trigone and posterior wall injections
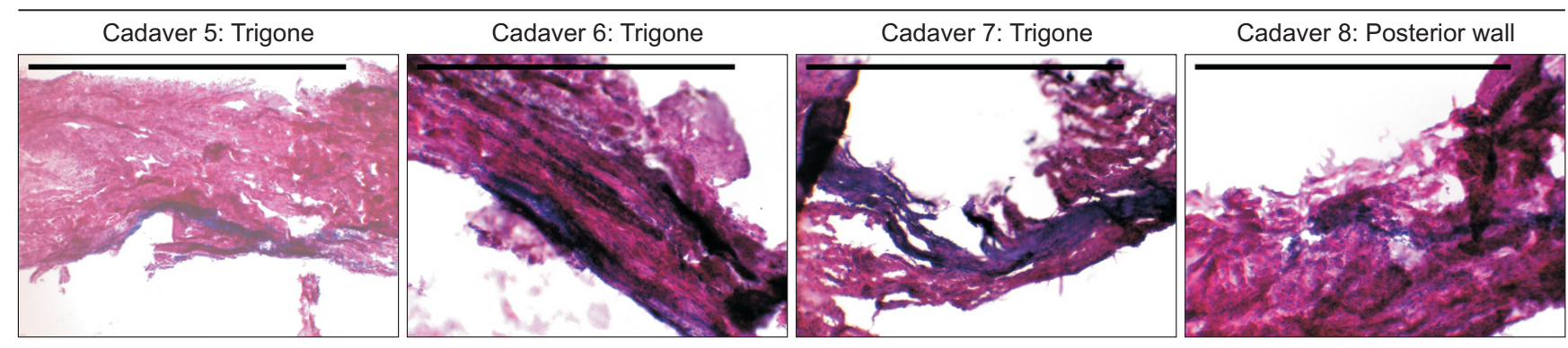

Fig. 2. Histologic analysis of bladder specimens (scale bar=1,000 $\mu \mathrm{m}$ ).

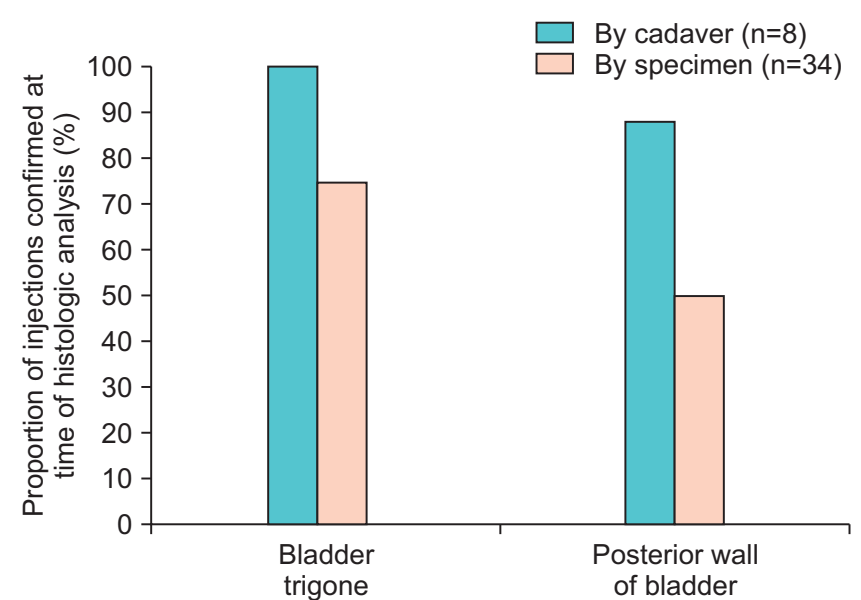

Fig. 3. Accuracy of surgeon's perception in targeting the detrusor layer under ultrasound guidance.

targeting this area we expect the same benefit of clinical efficacy compared to the traditional cystoscopic route. In addition, by performing 5 injections rather than the traditional 10-20 injections via the cystoscopic route, patient tolerability due to reduced number of injections (and thereby associated decrease in time to perform the procedure) may be improved with this technique. Therefore, this novel approach to intradetrusor chemodenervation could prove to be promising in human subjects.

Patient-related factors such as advanced age, bladder dysfunction and high detrusor pressures can increase bladder wall thickness at the trigone and the dome of the bladder [15]. Sonography (perineal, abdominal or transvaginal) has demonstrated variability in the anatomic measurements of the bladder [16]. Bladder wall thickness averages $1.2-1.9 \mathrm{~mm}$ when the bladder is filled with $200-250$ $\mathrm{mL}$ of fluid as assessed by abdominal ultrasound [17,18], compared to $4-5 \mathrm{~mm}$ by transvaginal ultrasound [19]. These results are similar to our finding of a mean posterior wall thickness of $4.0 \mathrm{~mm}$. It has also been shown that bladder neck thickness is greater than other areas of the bladder wall, with a reported mean thickness of $8.12-9.32 \mathrm{~mm}$ [20], consistent with our finding of a mean bladder neck thickness of $8.2 \mathrm{~mm}$ in the cadaver. With respect to these anatomic landmarks, the bladder anatomy of the cadavers used in this study reflects measurements of that seen in live subjects. There is a paucity of published data to compare with our measured mean interureteric distance of $24.4 \mathrm{~mm}$.

Our study demonstrates that this technique in cadavers accurately targets the bladder detrusor muscle in $64.7 \%$ of injections. This raises a potential concern for decreased effectiveness with this therapy and possible increased risk of adverse events associated with BTX injected into the wrong target. However, injections via the traditional cystoscopic route have been shown to have some degree of inaccuracy as well. Alsinnawi et al. [21] showed that, on magnetic resonance imaging following BTX bladder injections, there was considerable diffusion noted following injection therapy, where $80 \%$ of patients had contrast in the perivesical space. Another group found that $17.6 \%$ of contrast medium was within the peritoneal fat tissue [22]; however, no systemic side effects were observed. These findings may reflect 
diffusion of therapy following injection that is safe, with a low risk of adverse events.

The utility of cadavers for pre-clinical testing of novel procedures is well-established [23-25]. The cadaver gives the clinician a training platform on which to optimize technique and identify challenges with positioning and/or equipment prior to involving a live study subject. Using this approach, our study confirms appropriate depth and diffusion of injection as confirmed by subsequent histology [26], which would be neither practical nor ethical in live subjects.

Limitations of our approach need to be considered prior to translating cadaveric findings to live subjects. There may be significant differences between cadavers and live subjects, potentially related to both the advanced age of our cadavers (though by study design, age at time of death was not available to us for patient health protected purposes) and difference in quality of cadaveric tissue, such as bladder fibrosis. This may partially explain why, despite clear ultrasound guidance, overall accuracy of targeting the detrusor layer was less than $100.0 \%$. Our study adds to the growing body of literature in support of the potential utility of targeted injections into the trigone of the bladder. It would otherwise not be feasible to cystoscopically confirm targeting of the detrusor layer using concurrent cystoscopy in awake human subjects while performing transvaginal injection, given the close proximity of the urethra and vagina. Our next step will be to perform the transvaginal ultrasound guided technique in living human subjects and assess the efficacy of BTX intradetrusor injection for the treatment of refractory OAB. To avoid instrumentation of the urinary tract in live human subjects but allow adequate visualization of the urinary bladder by transvaginal ultrasound, patients will be encouraged to limit voiding for $1-2$ hours prior to the procedure. It is possible that variability in bladder volume may influence accuracy of traditional cystoscopic injections, however via the transvaginal route, we hypothesize that our technique of injection into the trigone and posterior wall may be less susceptible to bladder volume changes than injections into other areas of the bladder, such as the dome and lateral walls of the bladder.

Strengths of our study include the use of multiple cadavers with multiple [3-5] full thickness bladder biopsies in each cadaver, which confirmed the accurate targeting of the detrusor layer. The procedure was performed in the exact same sequence of steps with each cadaver, confirming the reproducibility of the technique between subjects. Our findings confirm that this technique is feasible and our histology supports that this approach has acceptable accuracy. Our next step will be to perform the transvaginal ultrasound guided technique in living human subjects and assess the efficacy of BTX intradetrusor injection for the treatment of refractory $\mathrm{OAB}$.

\section{CONCLUSIONS}

Intradetrusor injection of the bladder trigone and posterior wall under transvaginal ultrasound guidance is feasible and has acceptable accuracy.

\section{CONFLICTS OF INTEREST}

The authors have nothing to disclose.

\section{ACKNOWLEDGMENTS}

This study was Funded by The Society of Urodynamics, Female Pelvic Medicine and Urogenital Reconstruction Foundation Study of Chemodenervation 2017.

The project described herein was conducted with author (ADD) support from the KL2 component of the Stanford Clinical and Translational Science Award to Spectrum (NIH KL2 TR 001083).

\section{REFERENCES}

1. Sun Y, Luo D, Tang C, Yang L, Shen H. The safety and efficiency of onabotulinumtoxinA for the treatment of overactive bladder: a systematic review and meta-analysis. 2015;47:177988.

2. Gormley EA, Lightner DJ, Burgio KL, Chai TC, Clemens JQ, Culkin DJ, et al. Diagnosis and treatment of overactive bladder (non-neurogenic) in adults: AUA/SUFU guideline. J Urol 2012;188(6 Suppl):2455-63.

3. Nitti VW, Dmochowski R, Herschorn S, Sand P, Thompson C, Nardo C; EMBARK Study Group, et al. OnabotulinumtoxinA for the treatment of patients with overactive bladder and urinary incontinence: results of a phase 3, randomized, placebo controlled trial. J Urol 2013;189:2186-93.

4. Abdel-Meguid TA. Botulinum toxin-A injections into neurogenic overactive bladder--to include or exclude the trigone? A prospective, randomized, controlled trial. J Urol 2010;184:2423-8.

5. Kuo HC. Bladder base/trigone injection is safe and as effective as bladder body injection of onabotulinumtoxinA for idiopathic detrusor overactivity refractory to antimuscarinics. Neurourol Urodyn 2011;30:1242-8.

6. Lucioni A, Rapp DE, Gong EM, Fedunok P, Bales GT. Intra- 
vesical botulinum type A toxin injection in patients with overactive bladder: trigone versus trigone-sparing injection. Can J Urol 2006;13:3291-5.

7. Manecksha RP, Cullen IM, Ahmad S, McNeill G, Flynn R, McDermott TE, et al. Prospective randomised controlled trial comparing trigone-sparing versus trigone-including intradetrusor injection of abobotulinumtoxinA for refractory idiopathic detrusor overactivity. Eur Urol 2012;61:928-35.

8. Dobberfuhl AD, Chen B, Han X, Diaz E, Comiter CV. Ambulatory and cystometric response following single versus multiple onabotulinumtoxina detrusor injections in a rat model of overactive bladder induced by intravesical acetic acid [abstract]. Society of Urodynamics, Female Pelvic Medicine \& Urogenital Reconstruction 2017 winter meeting program book 2017. p. 147.

9. Dobberfuhl AD, Chen B, Han X, Diaz EC, Comiter C. No Adverse change in ambulatory voiding function after trigone onabotulinumtoxinA detrusor injection in the rat [abstract]. International Continence Society 2017 scientific programme. 2017. Abstract No. 775.

10. Pinto R, Costa D, Morgado A, Pereira P, Silva J, Cruz F. Results from a randomized, double blind, placebo-controlled trial to evaluate efficacy and safety of intra-trigonal injection of onabotulinum toxin A in patients with bladder pain syndrome/ interstitial cystitis [abstract]. J Urol 2017;197 Suppl 4:e46. Abstract No. PD01-01.

11. Stav K, Leibovici D, Goren E, Livshitz A, Siegel YI, Lindner A, et al. Adverse effects of cystoscopy and its impact on patients' quality of life and sexual performance. Isr Med Assoc J 2004;6:474-8.

12. Rahnama'i MS, Marcelissen TAT, Brierley B, Schurch B, de Vries P. Long-term compliance and results of intravesical botulinum toxin A injections in male patients. Neurourol Urodyn 2017;36:1855-9.

13. Bartley J, Han E, Gupta P, Gaines N, Killinger KA, Boura JA, et al. Transvaginal trigger point injections improve pain scores in women with pelvic floor hypertonicity and pelvic pain conditions. Female Pelvic Med Reconstr Surg 2018 Apr 4 [Epub]. http://doi.org/10.1097/SPV.0000000000000581.

14. Spradling K, Khoyilar C, Abedi G, Okhunov Z, Wikenheiser J, Yoon $\mathrm{R}$, et al. Redefining the autonomic nerve distribution of the bladder using 3-dimensional image reconstruction. J Urol 2015;194:1661-7.

15. Yang JM, Huang WC. Bladder wall thickness on ultrasono- graphic cystourethrography: affecting factors and their implications. J Ultrasound Med 2003;22:777-82.

16. Kuhn A, Bank S, Robinson D, Klimek M, Kuhn P, Raio L. How should bladder wall thickness be measured? A comparison of vaginal, perineal and abdominal ultrasound. Neurourol Urodyn 2010;29:1393-6.

17. Blatt AH, Titus J, Chan L. Ultrasound measurement of bladder wall thickness in the assessment of voiding dysfunction. J Urol 2008;179:2275-8; discussion 2278-9.

18. Oelke M, Höfner K, Jonas U, Ubbink D, de la Rosette J, Wijkstra H. Ultrasound measurement of detrusor wall thickness in healthy adults. Neurourol Urodyn 2006;25:308-17; discussion 318.

19. Khullar V, Salvatore S, Cardozo L, Bourne TH, Abbott D, Kelleher C. A novel technique for measuring bladder wall thickness in women using transvaginal ultrasound. Ultrasound Obstet Gynecol 1994;4:220-3.

20. Kuo HC. Measurement of detrusor wall thickness in women with overactive bladder by transvaginal and transabdominal sonography. Int Urogynecol J Pelvic Floor Dysfunct 2009;20:1293-9.

21. Alsinnawi M, Torreggiani W, Sheikh M, Thomas A, Donnellan J, Flynn R, et al. Delayed contrast-enhanced MRI to localize Botox after cystoscopic intravesical injection. Int Urol Nephrol 2015;47:893-8.

22. Mehnert U, Boy S, Schmid M, Reitz A, von Hessling A, Hodler J, et al. A morphological evaluation of botulinum neurotoxin A injections into the detrusor muscle using magnetic resonance imaging. World J Urol 2009;27:397-403.

23. Sermus LA, Sala-Blanch X, McDonnell G, Lobo CA, Nicholls BJ, van Geffen GJ, et al. Ultrasound-guided approach to nerves (direct vs. tangential) and the incidence of intraneural injection: a cadaveric study. Anaesthesia 2017;72:461-9.

24. Choquet O, Morau D, Biboulet P, Capdevila X. Where should the tip of the needle be located in ultrasound-guided peripheral nerve blocks? Curr Opin Anaesthesiol 2012;25:596-602.

25. Chuan A, Lim YC, Aneja H, Duce NA, Appleyard R, Forrest $\mathrm{K}$, et al. A randomised controlled trial comparing meat-based with human cadaveric models for teaching ultrasound-guided regional anaesthesia. Anaesthesia 2016;71:921-9.

26. Gupta P, Ehlert M, Sirls LT, Peters K. Transvaginal pelvic floor muscle injection technique: a cadaver study. Female Pelvic Med Reconstr Surg 2017;23:61-3. 